\title{
AUTOMORPHISMS OF THE DIMENSION GROUP AND GYRATION NUMBERS
}

\author{
K. H. KIM, F. W. ROUSH, AND J. B. WAGONER
}

\section{CONTENTS}

0 . Introduction

1. The sign-gyration-compatibility-condition

2. Gyration and orbit-sign numbers

3. Construction of $\operatorname{sgcc}_{A, m}$

4. Application of $\operatorname{sgcc}_{A, m}$

\section{SUMMARY}

Let $\operatorname{Aut}\left(\sigma_{A}\right)$ denote the group of automorphisms of a subshift of finite type $\left(X_{A}, \sigma_{A}\right)$ built from a primitive matrix $A$. We show that the sign-gyrationcompatibility-condition homomorphism $\mathrm{SGCC}_{A, m}$ defined on $\operatorname{Aut}\left(\sigma_{A}\right)$ factors through the group $\operatorname{Aut}\left(s_{A}\right)$ of automorphisms of the dimension group. This is used to find a mixing subshift of finite type with a permutation of fixed points that cannot be lifted to an automorphism of the shift. We also give an example of a mixing subshift of finite type where the dimension group representation is not surjective. This example is used in [KR3] to give examples of subshifts of finite type (reducible, with two mixing components) that are shift equivalent but not strong shift equivalent over the nonnegative integers.

\section{INTRODUCTION}

Methods of symbolic dynamics arise in such fields as information theory, ergodic theory and dynamical systems, cellular automata theory, and statistical mechanics. Among the simplest symbolic systems are the subshifts of finite type $\sigma_{A}: X_{A} \rightarrow X_{A}$ coming from square matrices $A$ with entries lying in the nonnegative integers $A^{+}$. Consult, for example, [DGS, E, Fr, PT]. In the setting of smooth dynamical systems, the matrix $A$ typically comes from a Markov partition on the zero-dimensional part of the nonwandering set of a diffeomorphism. There are many ways of choosing Markov partitions, and this produces different matrices $A$ so that the various shifts $\sigma_{A}: X_{A} \rightarrow X_{A}$ are topologically

Received by the editors November 11, 1990 and, in revised form, July 15, 1991.

1991 Mathematics Subject Classification. Primary 54H20, 57S99, 20F99; Secondary 60J10.

Key words and phrases. Sign-gyration-compatibility-condition homomorphism.

The first two authors were partially supported by NSF grant DMS 8820801 and the last was partially supported by NSF grant DMS 8801333 . 
equivalent. In fact, a central and still open problem is to find good methods for determining whether two subshifts of finite type $X_{A}$ and $X_{B}$ are indeed topologically equivalent.

One approach to this classification problem of subshifts of finite type (SFT's) was initiated by Williams in [Wi1, Wi2] and involves the notions of strong shift equivalence (SSE) and shift equivalence (SE). An elementary strong shift equivalence $(R, S): A \rightarrow B$ from $A$ to $B$ over $Z^{+}$consists of a pair of nonnegative integral matrices $R$ and $S$ such that $A=R S$ and $B=S R$. More generally, $A$ and $B$ are strong shift equivalent over $Z^{+}$iff there is a chain of elementary SSE's over $Z^{+}$from $A$ to $B$. Williams proved that $\left(X_{A}, \sigma_{A}\right)$ and $\left(X_{B}, \sigma_{B}\right)$ are topologically conjugate iff $A$ and $B$ are strong shift equivalent over $Z^{+}$. The problem is that SSE over $Z^{+}$is difficult to decide. A shift equivalence $R: A \rightarrow B$ over $Z^{+}$is a nonnegative integral matrix $R$ such that there is some nonnegative integral matrix $S$ and a positive integer $k$ satisfying $A R=R B$, $B S=S A, A^{k}=R S$, and $B^{k}=S R$. Shift equivalence over $Z^{+}$is easier to determine. In fact, it was proved in [KR1] that SE is decidable. SSE implies $\mathrm{SE}$, and Williams's influential work raised the now well-known question

(SHIFT)

Does SE imply SSE over over $Z^{+}$?

The nonnegativity condition is important, because it was shown by Effros and Williams that SE implies SSE over $Z$. See [BH, W4]. Using (4.1) of this paper, the first two authors [KR3] have recently given examples that now show the answer to SHIFT is negative in general. The examples of [KR3] are for reducible matrices $A$ and $B$. However, SHIFT is still open when $A$ and $B$ are primitive, i.e., when a power of $A$ and a power of $B$ have strictly positive entries. Some results are known for $2 \times 2$ matrices [B, CK].

A key ingredient in our present understanding of SFT's turns out to be their group of symmetries. The automorphism group $\operatorname{Aut}\left(\sigma_{A}\right)$ of $\left(X_{A}, \sigma_{A}\right)$ consists of those homeomorphisms of $X_{A}$ that commute with $\sigma_{A}$. Hedlund and coworkers [He] studied the automorphism groups of full Bernoulli shifts in the early 1960s. Then during the 1980 s there was a renewed interest in the groups $\operatorname{Aut}\left(\sigma_{A}\right)$, which are countable but are generally enormous. See [BLR]. Two useful representations of $\operatorname{Aut}\left(\sigma_{A}\right)$ have emerged: Krieger's dimension group representation [K, BLR] and the sign and gyration number homomorphisms of Boyle-Krieger [BK]. The first is essentially a matrix group representation and the latter go into finite cyclic groups. The main result (1.4) of this paper establishes a connection between these representations. The work of BoyleKrieger [BK] and of Fiebig [F1, F2] studies the fundamental "sign-gyrationcompatibility-condition," which is a relation between the sign numbers and the gyration numbers of certain automorphisms. We turn the relation into the "signgyration-compatibility-condition homomorphism" $\mathrm{SGCC}_{A, m}$ from $\operatorname{Aut}\left(\sigma_{A}\right)$ to the cyclic group $Z / m$ and show it factors through a homomorphism $\operatorname{sgcc}_{A, m}$ defined on the group $\operatorname{Aut}\left(s_{A}\right)$ of automorphisms of the dimension group. An explicit formula is given for $\operatorname{sgcc}_{A, 2}$ in (2.14) that is very useful for the applications mentioned in the Summary.

In $\S 1$ we define "sign-gyration-compatibility-condition homomorphism." The proof of (1.4) is given in $\S \S 2$ and 3. The construction of $\operatorname{sgcc}_{A, m}$ uses 
the algebraic topology framework for studying Aut $\left(\sigma_{A}\right)$ developed in [W1-W4] together with the positivity and localization methods of [KR2], the first step of which is the eventual positivity theorem of Handelman ([H, Theorem 2.3], see (3.4) below). All that is required to understand the examples in $\S 4$ is the statement (1.4) and the explicit polynomial formula (2.14) for $\operatorname{sgcc}_{A, 2}$. Our proof will show that primitive matrices shift equivalent over $\mathrm{Z}$ to the matrices in examples (4.1) and (4.3) also have, respectively, nonsurjectivity of the dimension group representation and a permutation of fixed points that does not lift to an automorphism of the shift.

\section{THE SIGN-GYRATION-COMPATIBILITY-CONDITION}

For $m \geq 1$, let $\mathrm{GY}_{A, m}: \operatorname{Aut}\left(\sigma_{A}\right) \rightarrow Z / m$ and $\mathrm{OS}_{A, m}: \operatorname{Aut}\left(\sigma_{A}\right) \rightarrow Z / 2$ be the dynamically defined gyration number and orbit sign number homomorphisms, respectively, introduced by Boyle-Krieger in [BK]. The definitions of $\mathrm{GY}_{A, m}$ and $\mathrm{OS}_{A, m}$ are repeated and generalized slightly in (2.3) and (2.4). When $m$ is even, we will consider $\operatorname{OS}_{A, m}$ as a homomorphism into $Z / m$ by viewing $Z / 2$ as the subgroup $\{0, m / 2\}$. For $m \geq 2$, consider the signgyration-compatibility-condition homomorphism $\operatorname{SGCC}_{A, m}: \operatorname{Aut}\left(\sigma_{A}\right) \rightarrow Z / m$ given by the formula

$$
\operatorname{SGCC}_{A, m}(\alpha)=\mathrm{GY}_{A, m}(\alpha)+\sum_{i} \mathrm{OS}_{A, m / 2^{i}}(\alpha)
$$

where $i>0$ and $\operatorname{OS}_{A, m / 2^{i}}(\alpha)$ is taken to be zero whenever $m / 2^{i}$ is not integral. In particular, $\operatorname{SGCC}_{A, m}(\alpha)=\mathrm{GY}_{A, m}(\alpha)$ if $m$ is odd. When $m=2$, we have

$$
\operatorname{SGCC}_{A, 2}(\alpha)=\mathrm{GY}_{A, 2}(\alpha)+\operatorname{OS}_{A, 1}(\alpha)
$$

A basic fact expressed by the sign-gyration-compatibility-condition theorems in $[\mathrm{BK}, \mathrm{N}]$ is

$$
\operatorname{SGCC}_{A, m}(\alpha)=0 \text { if } \alpha \text { is a product of simple automorphisms. }
$$

Fiebig showed (1.3) was valid for finite order elements $\alpha$ in the kernel $\operatorname{Aut}_{0}\left(\sigma_{A}\right)$ of the dimension group representation $\delta_{A}: \operatorname{Aut}\left(\sigma_{A}\right) \rightarrow \operatorname{Aut}\left(s_{A}\right)$ and it was finally proved in [KR2] that (1.3) holds for all $\alpha$ in Aut $_{0}\left(\sigma_{A}\right)$. A consequence is that $\operatorname{SGCC}_{A, m}(\alpha)$ only depends on its image $\delta_{A}(\alpha)$ in $\operatorname{Aut}\left(s_{A}\right)$. Our main result says that $\operatorname{SGCC}_{A, m}$ actually factors through $\operatorname{Aut}\left(s_{A}\right)$.

(1.4) Theorem. Let $A$ be a square integral matrix that is conjugate over the integers to an eventually positive integral matrix. There is a homomorphism $\operatorname{sgcc}_{A, m}: \operatorname{Aut}\left(s_{A}\right) \rightarrow Z / m$ with the property that if $A$ is nonnegative and aperiodic, then $\mathrm{SGCC}_{A, m}=\operatorname{sgcc}_{A, m} \cdot \delta_{A}$.

For a nonnegative square integral matrix $A, \operatorname{Aut}\left(s_{A}\right)$ is usually taken to be the group of automorphisms of the dimension group $G_{A}$ that commute with $s_{A}$ and preserve the order structure on $G_{A}$. See [BLR]. In the statement of (1.4) and throughout this paper, we let $\operatorname{Aut}\left(s_{A}\right)$ denote the larger group of 
automorphisms of $G_{A}$ that commute with $s_{A}$. We do not require that order be preserved even when $A$ is nonnegative.

\section{GYRATION AND ORBIT-SIGN NUMBERS}

To start, let $A$ denote a nonnegative square integral matrix and let $\left(X_{A}, \sigma_{A}\right)$ be the subshift of finite type associated to $A$. From [W3] we know there is an isomorphism

$$
\operatorname{Aut}\left(\sigma_{A}\right) \bmod \operatorname{Simp}\left(\sigma_{A}\right) \approx \pi_{1}\left(R S\left(Z^{+}\right), A\right),
$$

where $\operatorname{Simp}\left(\sigma_{A}\right)$ denotes Nasu's group of simple automorphisms. In view of (1.3) we therefore know that $\operatorname{SGCC}_{A, m}$ is defined on $\pi_{1}\left(R S\left(Z^{+}\right), A\right)$. An element $\gamma$ of $\pi_{1}\left(R S\left(Z^{+}\right), A\right)$ is represented by a loop

$$
\gamma=\prod_{i} \gamma\left(R_{i}, S_{i}\right)^{\epsilon_{i}}
$$

based at $A$ where $\epsilon_{i}= \pm 1$ and $\left(R_{i}, S_{i}\right): P(i-1) \rightarrow P(i)$ or $\left(R_{i}, S_{i}\right): P(i) \rightarrow$ $P(i-1)$ depending on whether $\epsilon_{i}=+1$ or $\epsilon_{i}=-1$, respectively. Our first goal will be to understand how to compute $\operatorname{SGCC}_{A}(\gamma)$ in terms of gyration and orbit sign numbers associated to each nonnegative elementary strong shift equivalence $\left(R_{i}, S_{i}\right)$. Then we recall from [W4] that $\operatorname{Aut}\left(s_{A}\right) \approx \pi_{1}(R S(Z), A)$ for a general matrix $A$ as in (1.4) and will use this equation in $\S 3$ to define the required homomorphism $\operatorname{sgcc}_{A}$ on $\pi_{1}(R S(Z), A)$ via the positivity and localization methods [KR2].

(2.2) Definition. Let $m \geq 1$. An m-basis $\mu$ for $\left(X_{A}, \sigma_{A}\right)$ consists of the choice of (a) an ordering $O_{1}, \ldots, O_{N}$ for all the orbits of periods dividing $m$ and (b) a choice of a base point $b_{i}$ on each orbit $O_{i}$.

Actually, we will only need to look at the orbits of periods $m / 2^{i}$ where $i \geq 0$. Let $\alpha:\left(X_{A}, \sigma_{A}\right) \rightarrow\left(X_{B}, \sigma_{B}\right)$ be a conjugacy between SFT's, and let $\mu$ and $\nu$ be $m$-bases, respectively, for $\left(X_{A}, \sigma_{A}\right)$ and $\left(X_{B}, \sigma_{B}\right)$. Generalizing [BK], we define the gyration number $\mathrm{GY}_{\mu \nu, m}(\alpha)$ in $Z / m$ and the orbit sign number $\mathrm{OS}_{\mu \nu, k}(\alpha)$ in $Z / 2$ for $k$ dividing $m$ as follows:

(2.3) $\operatorname{OS}_{\mu \nu, k}(\alpha)=$ sign of the bijection between the period $k$ orbits of

$$
\left(X_{A}, \sigma_{A}\right) \text { and those of }\left(X_{B}, \sigma_{B}\right) \text { induced by } \alpha \text {. }
$$

Let $O_{i}$ be a period $m$ orbit of $\left(X_{A}, \sigma_{A}\right)$, and let $O_{j}=\alpha\left(O_{i}\right)$ denote the period $m$ orbit of $\left(X_{B}, \sigma_{B}\right)$ which is its image by $\alpha$. There is an integer $r=r_{i}$, uniquely determined modulo $m$, so that $\alpha\left(b_{i}\right)=\sigma^{r}\left(b_{j}\right)$ where $\sigma=\sigma_{B}$. Define

$$
\mathrm{GY}_{\mu \nu, m}(\alpha)=\sum_{i} r_{i} \text { modulo } m
$$

Finally, we let

$$
\operatorname{SGCC}_{\mu \nu, m}(\alpha)=\mathrm{GY}_{\mu \nu, m}(\alpha)+\sum_{i} \mathrm{OS}_{\mu \nu, m / 2^{i}}(\alpha) \quad \text { for } i>0 .
$$


The standard Boyle-Krieger gyration and orbit sign homomorphisms $\mathrm{GY}_{A, m}$ and $\mathrm{OS}_{A, k}$ and the homomorphism $\mathrm{SGCC}_{A, m}$ are obtained by taking $A=B$ and $\mu=\nu$. In particular, they do not depend on the choice of $\mu$. See [BK]. However, the quantities $\mathrm{GY}_{\mu \nu, m}, \mathrm{OS}_{\mu \nu, k}$, and $\mathrm{SGCC}_{\mu \nu, m}$ do depend very much on the choice of $m$-bases $\mu$ and $\nu$ when $A \neq B$. Nevertheless, they are useful in constructing $\operatorname{sgcc}_{A, m}$ where we will take certain natural choices of $m$-bases coming from lexicographical ordering.

(2.6) Proposition. Let $\alpha:\left(X_{A}, \sigma_{A}\right) \rightarrow\left(X_{B}, \sigma_{B}\right)$ and $\beta:\left(X_{B}, \sigma_{B}\right) \rightarrow\left(X_{C}, \sigma_{C}\right)$ be conjugacies, and choose $m$-bases $\mu, \nu$, and $\eta$ for $\left(X_{A}, \sigma_{A}\right),\left(X_{B}, \sigma_{B}\right)$, and $\left(X_{C}, \sigma_{C}\right)$, respectively. Then

$$
\begin{aligned}
\mathrm{GY}_{\mu \eta, m}(\alpha \beta) & =\mathrm{GY}_{\mu \nu, m}(\alpha)+\mathrm{GY}_{\nu \eta, m}(\beta), \\
\mathrm{OS}_{\mu \eta, k}(\alpha \beta) & =\operatorname{OS}_{\mu \nu, k}(\alpha)+\operatorname{OS}_{\nu \eta, k}(\beta) \quad \text { for } k \text { dividing } m .
\end{aligned}
$$

Consequently

$$
\operatorname{SGCC}_{\mu \eta, m}(\alpha \beta)=\operatorname{SGCC}_{\mu \nu, m}(\alpha)+\operatorname{SGCC}_{\nu \eta, m}(\beta) .
$$

The proof follows easily from the definitions and is entirely similar to the one showing that $\mathrm{GY}_{A, m}$ and $\mathrm{OS}_{A, k}$ are homomorphisms.

The next step in constructing $\operatorname{sgcc}_{A, m}$ for $A$ as in (1.4) is to discuss the gyration and orbit sign numbers of an elementary strong shift equivalence $(R, S)$ : $P \rightarrow Q$ over the nonnegative integers. Starting with a nonnegative integral matrix $P$ on a set of states $\mathscr{I}$, the standard edge path construction produces a SFT $\left(X_{P^{\prime}}, \sigma_{P^{\prime}},\right)$ where $P^{\prime}$ is a zero-one matrix whose states $\mathscr{S}^{\prime}$ are edges $e=(i, a, j)$ from $i$ to $j$ in $\mathscr{I}$ with $1 \leq a \leq P(i, j)$ and $P^{\prime}(e, f)=1$ iff the end state of $e$ is the initial state of $f$. As in (2.1) of [W3] bipartite coding gives a certain elementary strong shift equivalence $\left(R^{\prime}, S^{\prime}\right): P^{\prime} \rightarrow Q^{\prime}$ of zeroone matrices determined up to composition with simple automorphisms on the right and left. Choose $m$-bases $\mu$ for $\left(X_{P^{\prime}}, \sigma_{P^{\prime}}\right)$ and $\nu$ for $\left(X_{Q^{\prime}}, \sigma_{Q^{\prime}}\right)$. We then define

$$
\operatorname{SGCC}_{m}(R, S)=\operatorname{SGCC}_{\mu \nu, m}\left(R^{\prime}, S^{\prime}\right)
$$

and see from (1.3) that this does not depend upon the choice of $\left(R^{\prime}, S^{\prime}\right)$, although it does depend upon $\mu$ and $\nu$. For simplicity of notation, we will usually leave off the subscript $\mu \nu$ on the left-hand side of (2.7). We define $\mathrm{OS}_{k}(R, S)$ and $\mathrm{GY}_{m}(R, S)$ similarly.

Consider the path $\gamma$ in $R S\left(Z^{+}\right)$as in (2.1), but not necessarily a closed loop. Let $\alpha$ be the corresponding composition

$$
\alpha=\prod_{i} c\left(R_{i}, S_{i}\right)^{\epsilon_{i}}
$$

of elementary conjugacies where $\epsilon_{i}= \pm 1$. Make a choice of an $m$-basis $\mu_{i}$ for each subshift of finite type $\left(X_{P(i)}, \sigma_{P(i)}\right)$ in the chain, and define each $\operatorname{SGCC}_{m}\left(R_{i}, S_{i}\right)$ accordingly as in (2.7). 
(2.9) Proposition. If $\gamma$ is a closed loop in $R S\left(Z^{+}\right)$starting and ending at $A$, then

$$
\operatorname{SGCC}_{A, m}(\alpha)=\sum_{i} \epsilon_{i} \operatorname{SGCC}_{m}\left(R_{i}, S_{i}\right)
$$

In particular,

$$
\sum_{i} \epsilon_{i} \mathrm{SGCC}_{m}\left(R_{i}, S_{i}\right)
$$

is independent of the choice of $m$-basis $\mu_{i}$ at each vertex $P(i)$.

Proof. $\mathrm{SGCC}_{m}$ applied to the elementary conjugacy $c\left(R_{i}, S_{i}\right)^{\epsilon_{i}}$ is equal to $\epsilon_{i} \mathrm{SGCC}_{m}\left(R_{i}, S_{i}\right)$. So the equation in (2.9) follows directly from (2.8) and the composition formulas in (2.6). Consequently, the right-hand side does not depend on the choices of $m$-bases. Q.E.D.

In our situation, a convenient choice for an $m$-basis will come from a linear ordering of the vertices and a lexicographical ordering of the edges in the graph associated to a nonnegative integral matrix. Let $M: \mathscr{I} \times \mathscr{K} \rightarrow Z^{+}$be any nonnegative integral matrix where each of the sets $\mathscr{I}$ and $\mathscr{K}$ are linearly ordered. Let $e=(i, a, k)$ be an edge from $i$ to $k$ in the graph for $M$ where $1 \leq a \leq M_{i k}$. Similarly, let $f=(j, b, l)$ be an edge from $j$ to $l$ where $1 \leq b \leq M_{j l}$. The standard ordering of edges of $M$ defines $e<f$ iff

$$
\begin{gathered}
(i, k)<(j, l) \quad \text { by left lexicographical ordering of } \mathscr{I} \times \mathscr{K} \text {, or } \\
(i, k)=(j, l) \text { and } a<b .
\end{gathered}
$$

Let $P: \mathscr{I} \times \mathscr{I} \rightarrow Z^{+}$be any nonnegative integral matrix. A periodic point of $\left(X_{P^{\prime}}, \sigma_{P^{\prime}}\right)$ of period dividing $m$ is given by a pair $\{i, p\}$ where $i=\left(i_{0}, \ldots, i_{m}\right)$ is a sequence of vertices in the graph for $P$ with $i_{0}=i_{m}$ and $p=\left(p_{1}, \ldots, p_{m}\right)$ is a sequence of edges in the graph where $p_{\alpha}$ goes from $i_{\alpha-1}$ to $i_{\alpha}$. Make a choice

$$
\text { of a linear ordering of the set of vertices } \mathscr{I} \text {. }
$$

The sequences $i=\left(i_{0}, \ldots, i_{m}\right)$ and $p=\left(p_{1}, \ldots, p_{m}\right)$ can then be ordered lexicographically from the left, and we order the periodic points lexicographically from the left by setting

$$
\{i, p\}<\{j, q\} \quad \text { iff } i<j \quad \text { or } \quad i=j \text { and } p<q .
$$

(2.13) Definition. An $m$-basis for $\left(X_{A}, \sigma_{A}\right)$ with the standard lexicographical ordering is obtained by taking the reference point on each orbit with period dividing $m$ to be the periodic point of least lexicographical order on that orbit and then ordering the orbits by the lexicographical order of these reference points.

Let $(R, S): P \rightarrow Q$ be an elementary strong shift equivalence over $Z^{+}$. Let $\operatorname{SGCC}_{2}(R, S)$ be computed dynamically as in (2.5) and (2.7) with respect to standard lexicographical 2-bases for $\left(X_{P}, \sigma_{P}\right)$ and $\left(X_{Q}, \sigma_{Q}\right)$.

(2.14) Proposition. The value of $\operatorname{SGCC}_{2}(R, S)$ is given by the algebraic formula

$$
\operatorname{SGCC}_{2}(R, S)=\sum_{\substack{i<j \\
k>l}} R_{i k} S_{k i} R_{j l} S_{l j}+\sum_{\substack{i<j \\
k \geq l}} R_{i k} S_{k j} R_{j l} S_{l i}+\sum_{i, k}\left(\begin{array}{c}
R_{i k} \\
2
\end{array}\right)\left(S_{k i}\right)^{2}
$$


modulo 2 where $\left(\begin{array}{c}R_{i k} \\ 2\end{array}\right)=R_{i k}\left(R_{i k}-1\right) / 2$ is the binomial coefficient of $R_{i k}$ items taken two at a time.

Before starting on the proof of (2.14) we will discuss the three main steps in computing $\mathrm{SGCC}_{m}(R, S)$ using $m$-bases with the standard lexicographical ordering (2.13). This is also needed for the construction of $\operatorname{sgcc}_{m}$ in $\S 3$.

Let $P: \mathscr{I} \times \mathscr{I} \rightarrow Z^{+}$and $Q: \mathscr{K} \times \mathscr{K} \rightarrow Z^{+}$be a nonnegative integral matrices where $\mathscr{I}$ and $\mathscr{K}$ are linearly ordered. First we recall more explicitly how a strong shift equivalence $(R, S): P \rightarrow Q$ over $Z^{+}$gives rise to a bipartite code $\left(R^{\prime}, S^{\prime}\right): P^{\prime} \rightarrow Q^{\prime}$ by way of two bijections $\mathfrak{p}: P \approx R S$ and $\mathfrak{q}: Q \approx S R$ as in [W3, 2.5]. Suppose $P$ is an $(m \times m)$-matrix and $Q$ is an $(n \times n)$-matrix.

Fix a pair of vertices $(i, j)$ of $P$ and consider an entry $P_{i j}$ in $P$. Use the equation $P=R S$ to write

$$
P_{i j}=R_{i 1} S_{1 j}+\cdots+R_{i k} S_{k j}+\cdots+R_{i n} S_{n j},
$$

where the terms on the right are ordered increasingly in accordance with the given ordering of the states $k$ of $Q$ as chosen in (2.11). Let $C_{0}=0$ and for each $1 \leq k \leq n-1$, let

$$
C_{k}=R_{i 1} S_{1 j}+\cdots+R_{i k} S_{k j}
$$

Then choose $\mathfrak{p}: P \approx R S$ so that for each $1 \leq k \leq n, \mathfrak{p}$ is the bijection between the set of edges

$$
\left\{(i, p, j) \mid C_{k-1}<p \leq C_{k-1}+R_{1 k} S_{k j}\right\}
$$

and the set of pairs of edges

$$
\left\{((i, r, k),(k, s, j)) \mid 1 \leq r \leq R_{i k} \text { and } 1 \leq s \leq S_{k j}\right\}
$$

defined by the formula

$$
p=C_{k-1}+(r-1) S_{k j}+s .
$$

For each $k,(2.18)$ is an order-preserving bijection between the set (2.16) ordered linearly by the integers $p$ and the set (2.17) of pairs of integers $(r, s)$ ordered lexicographically from the left.

Fix a pair of vertices $(k, l)$ of $Q$ and consider an entry $Q_{k_{1}}$ in $Q$. Use the equation $Q=S R$ to write

$$
Q_{k l}=S_{k 1} R_{1 l}+\cdots+S_{k i} R_{i l}+\cdots+S_{k m} R_{m l},
$$

where the terms on the right are ordered increasingly in accordance with the given ordering of the states $i$ of $P$ as chosen in (2.11). Let $D_{0}=0$ and for each $1 \leq i \leq m-1$, let

$$
D_{i}=S_{k 1} R_{1 l}+\cdots+S_{k i} R_{i l} .
$$

Then choose $\mathfrak{q}: Q \approx S R$ so that for each $1 \leq i \leq m, \mathfrak{q}$ is the bijection between the set

$$
\left\{(k, q, l) \mid D_{i-1}<q \leq D_{i-1}+S_{k i} R_{i l}\right\}
$$


and the set

$$
\left.\{(k, s, i),(i, r, l),) \mid 1 \leq s \leq S_{k i} \text { and } 1 \leq r \leq R_{i l}\right\}
$$

defined by the formula

$$
q=D_{i-1}+(s-1) R_{i l}+r .
$$

For each $i,(2.22)$ is an order-preserving bijection between the set (2.20) ordered linearly by the integers $q$ and the set $(2.21)$ of pairs of integers $(s, r)$ ordered lexicographically from the left.

At this point we have a bijection $\mathfrak{p}(X)=U V$ between the (directed) edges $X=(i, p, j)$ from $i$ to $j$ in the graph of $P$ and pairs of edges $U V$ where $U=(i, r, k)$ is an edge from $i$ to $k$ in the graph of $R$ and $V=(k, s, j)$ is an edge from $k$ to $j$ in the graph of $S$. We will call $U$ the tail of $\mathfrak{p}(X)$ and $V$ the head of $\mathfrak{p}(X)$. In particular, the initial point of $U$ is $i$ and the final point of $U$ is $k$. The initial point of $V$ is $k$ and the final point of $V$ is $j$. Similarly, we have a bijection $\mathfrak{q}(Y)=V U$ where $Y=(k, q, l), V=(k, s, i)$ is the tail of $\mathfrak{q}(Y)$, and $U=(i, r, l)$ is the head of $\mathfrak{q}(Y)$. As in [W3], we then obtain the strong shift equivalence $P^{\prime}=R^{\prime} S^{\prime}$ and $Q^{\prime}=S^{\prime} R^{\prime}$ by letting

$$
\begin{array}{ll}
R^{\prime}(X, Y)=1 & \text { iff head of } \mathfrak{p}(X)=\text { tail of } \mathfrak{q}(Y), \\
S^{\prime}(Y, X)=1 & \text { iff head of } \mathfrak{q}(Y)=\text { tail of } \mathfrak{p}(X) .
\end{array}
$$

The resulting conjugacy $c\left(R^{\prime}, S^{\prime}\right):\left(X_{P^{\prime}}, \sigma_{P^{\prime}}\right), \rightarrow\left(X_{Q^{\prime}}, \sigma_{Q^{\prime}}\right)$ can then be described in three stages:

Step 1. The bijection $\mathfrak{p}$ takes

$$
\{i, p\} \rightarrow\{i, k, r, s\} \text {. }
$$

Here $i=\left(i_{0}, \ldots, i_{m}\right)$ where $i_{\alpha}$ is a vertex in the $P$-graph with $i_{0}=i_{m}$, $p=\left(p_{1}, \ldots, p_{m}\right)$ where $p_{\alpha}$ is an edge in the $P$-graph from $i_{\alpha-1}$ to $i_{\alpha}, k=$ $\left(k_{0}, \ldots, k_{m}\right)$ where $k_{\alpha}$ is a vertex in the $Q$-graph with $k_{0}=k_{m}, r=\left(r_{1}, \ldots\right.$, $\left.r_{m}\right)$ where $r_{\alpha}$ is an edge in the $R$-graph from $i_{\alpha-1}$ to $k_{\alpha-1}$, and $s=\left(s_{1}, \ldots, s_{m}\right)$ where $s_{\alpha}$ is an edge in the $S$-graph from $k_{\alpha-1}$ to $i_{\alpha}$.

Step. 2 The bijection $\mathfrak{r s}$ coming from (2.23) takes

$$
\{i, k, r, s\} \rightarrow\{k, i, s, \sigma(r)\}
$$

where $\sigma(r)=\left(r_{2}, \ldots, r_{m}, r_{1}\right)$. On occasion we will abbreviate (2.25) by writing

$$
\left|r_{1} s_{1}\right| r_{2} s_{2}|\cdots| r_{m} s_{m}|\rightarrow| s_{1} r_{2}\left|s_{2} r_{3}\right| \cdots\left|s_{m} r_{1}\right| \text {. }
$$

Step 3. The inverse of the bijection $q$ takes

$$
\{k, i, s, \sigma(r)\} \rightarrow\{k, q\},
$$

where $q=\left(q_{1}, \ldots, q_{m}\right)$ with $q_{\alpha}$ an edge in the $Q$-graph from $k_{\alpha-1}$ to $k_{\alpha}$. The quadruples $\{i, k, r, s\}$ and $\{k, i, s, \sigma(r)\}$ are ordered lexicographically from the left.

Now we can give the proof of (2.14).

Contribution of $\mathrm{OS}_{1}$. To compute the determinant of $\alpha=c\left(R^{\prime}, S^{\prime}\right)$ on fixed points, it suffices to count the number of pairs modulo 2 of fixed points $X$ and $X^{\prime}$ such that $X<X^{\prime}$, but where $Y=\alpha(X)$ and $Y^{\prime}=\alpha\left(X^{\prime}\right)$ satisfy $Y>Y^{\prime}$. The following diagram describes the procedure in $(2.23)$ : 

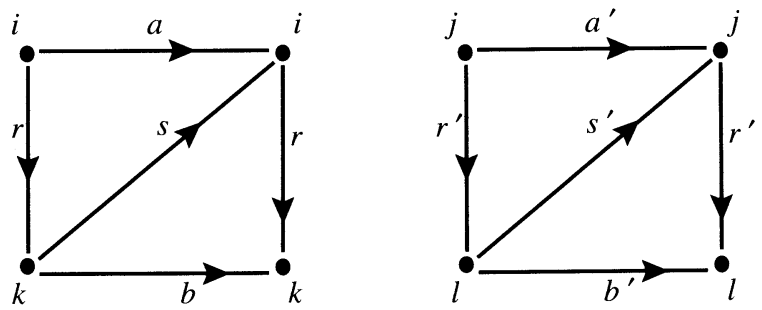

The edges in the diagram are labelled

$$
\begin{array}{ll}
X=(i, a, i), & X^{\prime}=\left(j, a^{\prime}, j\right), \\
U=(i, r, k), & U^{\prime}=\left(j, r^{\prime}, l\right), \\
V=(k, s, i), & V^{\prime}=\left(l, s^{\prime}, j\right), \\
Y=(k, b, k), & Y^{\prime}=\left(l, b^{\prime}, l\right) .
\end{array}
$$

There are only four cases where the values $i, j, k, l$ are such that a switch in the order of the fixed points might occur.

Case 1. $i<j$ and $k>l$. Then $X<X^{\prime}$ and $Y>Y^{\prime}$ for all relevant values of $a, b, r, s$, and $a^{\prime}, b^{\prime}, r^{\prime}, s^{\prime}$. The total number of switches is

$$
\sum_{i<j, k>l} R_{i k} S_{k i} R_{j l} S_{l j}
$$

Case 2. $i<j$ and $k=l$. Then $X<X^{\prime}$. We have $D_{i-1}<b \leq D_{i}$ and $D_{j-1}<b^{\prime} \leq D_{j}$. Since $i<j$, we must have $b<b^{\prime}$ and therefore $Y<Y^{\prime}$. No switches.

Case 3. $i=j$ and $k>l$. We have $C_{k-1}<a \leq C_{k}$ and $C_{l-1}<a^{\prime} \leq C_{l}$. The assumption $k>l$ implies $a>a^{\prime}$ and contradicts the assumption that $X<X^{\prime}$. So this case does not occur.

Case 4. $i=j$ and $k=l$. As in (2.26), $X=|r s|, Y=|s r|, X^{\prime}=\left|r^{\prime} s^{\prime}\right|$, and $Y^{\prime}=\left|s^{\prime} r^{\prime}\right|$. We must count the number of pairs $r s, r^{\prime} s^{\prime}$ such that $r s<r^{\prime} s^{\prime}$ but $s^{\prime} r^{\prime}<s r$.

(a) If $r<r^{\prime}$, then we must have $s^{\prime}<s$ in order to get a switch. The total number of switches is

$$
\sum_{i, k} \frac{R_{i k}\left(R_{i k}-1\right)}{2} \frac{S_{k i}\left(S_{k i}-1\right)}{2}
$$

(b) If $r=r^{\prime}$ but $s<s^{\prime}$, then $s r<s^{\prime} r^{\prime}$ and no switch occurs.

Contribution of $\mathrm{GY}_{2}$. Let $X$ be the base point on an orbit of period exactly 2 , and let $Y=\alpha(X)$ be its image under $\alpha=c\left(R^{\prime}, S^{\prime}\right)$. The following diagram describes the procedure in $(2.23)$ : 


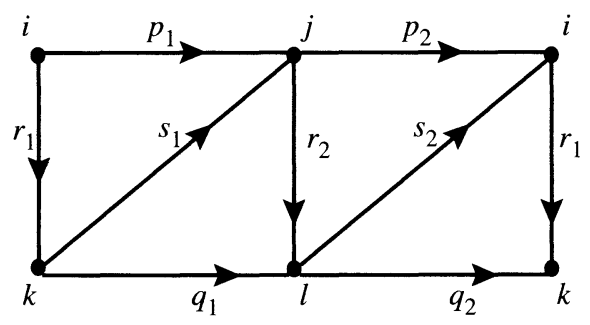

As in (2.26) we have $X=\left|r_{1} s_{1}\right| r_{2} s_{2} \mid$ and $Y=\left|s_{1} r_{2}\right| s_{2} r_{1} \mid$. To compute $\mathrm{GY}_{2}$ we must count the number of times that $\sigma_{B}(Y)=\left|s_{2} r_{1}\right| s_{1} r_{2} \mid$ is the base point on the orbit through $Y$; that is, we must compute the number of times where $Y>\sigma_{B}(Y)$. There are only four cases where the values $i, j, k, l$ are such that this might occur.

Case 1. $i<j$ and $k>l$. Then $Y>\sigma_{B}(Y)$ for all relevant values of $p_{1}, q_{1}, p_{2}$, and $q_{2}$. The total number of contributions to $\mathrm{GY}_{2}$ is

$$
\sum_{i<j, k>l} R_{i k} S_{k j} R_{j l} S_{l i} .
$$

Case 2. $i<j$ and $k=l$. We have $D_{j-1}<q_{1} \leq D_{j}$ and $D_{i-1}<q_{2} \leq D_{i}$. Since $i<j$, we must have $q_{2}<q_{1}$ and therefore $Y>\sigma_{B}(Y)$. The total number of switches is $\sum_{i<j, k=l} R_{i k} S_{k j} R_{j k} S_{k i}$.

Case 3. $i=j$ and $k>l$. We have $C_{k-1}<p_{1} \leq C_{k}$ and $C_{l-1}<p_{2} \leq C_{l}$. The assumption $k>l$ implies $p_{1}>p_{2}$ and contradicts the assumption that $X$ is the base point on its orbit. No contribution.

Case 4. $i=j$ and $k=l$. Since $X$ is the base point on the orbit, we have $r_{1} s_{1}<r_{2} s_{2}$ as left lexicographically ordered pairs.

(a) If $r_{1}<r_{2}$, then whenever $s_{2} \leq s_{1}$ we have $Y>\sigma_{B}(Y)$ and the number of contributions is

$$
\sum_{i, k} \frac{R_{i k}\left(R_{i k}-1\right)}{2} \frac{S_{k i}\left(S_{k i}+1\right)}{2} \text {. }
$$

(b) If $r_{1}=r_{2}$ but $s_{1}<s_{2}$, then $s_{1} r_{2}<s_{2} r_{1}$ as left lexicographically ordered pairs. So $Y<\sigma_{B}(Y)$. No contribution.

Summing up all these contributions completes the proof of (2.14). As an immediate consequence, we have

(2.28) Addition Lemma $(m=2)$. Let $(E, F): E F \rightarrow F E$ and $(G, H): G H \rightarrow$ $H G$ be elementary strong shift equivalences over $Z^{+}$. Assume the sizes of $E$ and $G$ and of $F$ and $H$ are the same. Let $a \equiv 0 \bmod 4$ and $b \equiv 0 \bmod 2$. Then

$$
\operatorname{SGCC}_{2}(E+a G, F+b H)=\operatorname{SGCC}_{2}(E, F)
$$

where

$$
\begin{aligned}
(E+a G, F+b H): & E F+a G F+b E H+a b G H \\
& \rightarrow F E+a F G+b H E+b a H G .
\end{aligned}
$$


(2.29) Remark. If the $R_{i k}$ and $S_{k j}$ are allowed to assume integer values, the algebraic formula in (2.14) still clearly satisfies the same addition property as in (2.28).

We next extend (2.28) to all $m \geq 2$.

(2.30) Addition Lemma $(m \geq 2)$. Let $(E, F): E F \rightarrow F E$ and $(G, H): G H \rightarrow$ $H G$ be elementary strong shift equivalences over $Z^{+}$. Assume the sizes of $E$ and $G$ and of $F$ and $H$ are the same. Let $a$ and $b$ be nonnegative integers that are congruent to zero modulo $m((2 m) !)$. Then

$$
\operatorname{SGCC}_{m}(E+a G, F+b H)=\operatorname{SGCC}_{m}(E, F) .
$$

This is an immediate consequence of the following proposition. Let $(R, S)$ : $P \rightarrow Q$ be a strong shift equivalence over $Z^{+}$where the states of $P$ and $Q$ are respectively linearly ordered sets $\mathscr{I}$ and $\mathscr{K}$.

(2.31) Proposition. Fix $m \geq 2$. There is an algebraic expression $\operatorname{sgc}_{m}(R, S)$ formed by taking a sum of certain products of binomial coefficients $\left(\begin{array}{l}L \\ d\end{array}\right)$ such that

(1) $L$ is either an entry $R_{i k}$ of $R$ or an entry $S_{k j}$ of $S$ and $1 \leq d \leq 2 m$;

(2) $\mathrm{sgc}_{m}$ depends only on $m$ and the choice of linear ordering for $\mathscr{I}$ and $\mathscr{K}$

(3) $\operatorname{SGCC}_{m}(R, S)=\operatorname{sgc}_{m}(R, S)$.

The remainder of this section concerns the proof of (2.31).

Let $f$ and $g$ be functions from a set $X$ to a linearly ordered set $\mathscr{L}$. We say $f$ and $g$ have the same order type provided that for any pair of elements $x$ and $y$ in $X$ we have

$$
\begin{aligned}
& f(x)=f(y) \quad \text { iff } g(x)=g(y), \\
& f(x)<f(y) \quad \text { iff } g(x)<g(y) .
\end{aligned}
$$

For example, let $f:\{1,2,3,4\} \rightarrow R$ be given by $f(1)=2, f(2)=10, f(3)$ $=5$, and $f(4)=2$. Then the set of all functions with the same order type as $f$ consists of those functions $g$ where $g(1)=g(4)<g(3)<g(2)$. If $X$ is finite and $\mathscr{L}$ is infinite, then the number of order types is finite and only depends on the cardinality of $X$. If $\beta: X \rightarrow X$ is a bijection, then $f$ and $g$ have the same order type iff $f \beta$ and $g \beta$ have the same order type. More generally, consider two sequences $\left\{f_{1}, \ldots, f_{q}\right\}$ and $\left\{g_{1}, \ldots, g_{q}\right\}$ where for each $1 \leq i \leq q$ both $f_{i}$ and $g_{i}$ are functions from a set $X_{i}$ to a linearly ordered set $\mathscr{L}_{i}$. We allow for the possibility that $X_{i} \neq X_{j}$ or $\mathscr{L}_{i} \neq \mathscr{L}_{j}$ when $i \neq j$. We say that these sequences have the same order type iff each of $f_{i}$ and $g_{i}$ have the same order type for $1 \leq i \leq q$.

Let $X=\{1,2, \ldots, n\}$ and let $\sigma: X \rightarrow X$ be the shift, i.e., $\sigma(i)=i-1$ $\bmod n$. Order the functions from $X$ to $\mathscr{L}$ lexicographically from the left. The basic observation underlying the proof of (2.31) is that, for any integer $k$, the order type of $f$ completely determines the order type of $f \sigma^{k}$ and whether $f=f \sigma^{k}$. Therefore the order type of $f$ determines 
(i) the exact period of any function $f: X \rightarrow \mathscr{L}$ under $\sigma$ (by definition, this period is the smallest $k$ such that $\left.f=f \sigma^{k}\right)$;

(ii) the unique number $k$ modulo the period of $f$ such that $g=f \sigma^{k}$ whenever $g$ lies in the $\sigma$-orbit of $f$.

Moreover, the period in (i) depends only on the order type of $f$ and the integer $k$ in (ii) depends only on the order types of $f$ and $g$. If the period of $f$ is exactly $p$, then there is a unique integer $k \bmod (p)$ so that $f \sigma^{k}$ is the function of least lexicographical order among functions in orbit of $f$ under $\sigma$. This $k$ will be called the rotation number of $f$. The formula for $\mathrm{sgc}_{m}$ in (2.31) will be obtained by summing up the contribution to the gyration and orbit sign over certain order types.

Let $M: \mathscr{C} \times \mathscr{D} \rightarrow R$ be a matrix where $\mathscr{C}$ and $\mathscr{D}$ are linearly ordered. The edges of $M$ will be given the standard ordering as in (2.10). The order type of a sequence $e=\left\{e_{1}, \ldots, e_{m}\right\}$ or edges will then be obtained by considering it to be a function from $\{1, \ldots, m\}$ into the set $\mathscr{C} \times \mathscr{D} \times R$ ordered lexicographically from the left. It will be convenient to denote an edge $\left(c_{\alpha-1}, e e_{\alpha}, d_{\alpha}\right)$ of $M$ where $1 \leq e e_{\alpha} \leq M\left(c_{\alpha-1}, d_{\alpha}\right)$ by the symbol $e_{\alpha}$.

Now let $(R, S): P \rightarrow Q$ be an elementary SSE over $Z^{+}$as in (2.31). Let $\{i, p\}$ and $\left\{i^{\prime}, p^{\prime}\right\}$ correspond to $\{i, k, r, s\}$ and $\left\{i^{\prime}, k^{\prime}, r^{\prime}, s^{\prime}\right\}$ as in (2.24). The sequences $i, i^{\prime}, k$, and $k^{\prime}$ have order types as functions from $\{0, \ldots, m\}$ to $\mathscr{I}$ or $\mathscr{K}$ considered as, say, imbedded in $R$, and the sequences or edges $p, p^{\prime}, r, s, r^{\prime}$, and $s^{\prime}$ have order types as explained just above.

(2.34) Lemma. Suppose $\{i, k, r, s\}$ and $\left\{i^{\prime}, k^{\prime}, r^{\prime}, s^{\prime}\right\}$ have the same order type. Then $\{i, p\}$ and $\left\{i^{\prime}, p^{\prime}\right\}$ have the same order type and so do $\{k, i, s$, $\sigma(r)\}$ and $\left\{k^{\prime}, i^{\prime}, s^{\prime}, \sigma\left(r^{\prime}\right)\right\}$. Similarly, if $\{k, i, s, r\}$ and $\left\{k^{\prime}, i^{\prime}, s^{\prime}, r^{\prime}\right\}$ have the same order type then so do the pairs $\{k, q\}$ and $\left\{k^{\prime}, q^{\prime}\right\}$.

Proof of (2.34). The quadruples $\{k, i, s, \sigma(r)\}$ and $\left\{k^{\prime}, i^{\prime}, s^{\prime}, \sigma\left(r^{\prime}\right)\right\}$ clearly have the same order type because $\sigma$ preserves order types. To show that $\{i, p\}$ and $\left\{i^{\prime}, p^{\prime}\right\}$ have the same order type, it suffices to show that $p$ and $p^{\prime}$ have the same order type. For any pair of indices $\alpha$ and $\beta$ we must show that
(A) $p_{\alpha}=p_{\beta}$ iff $p_{\alpha}^{\prime}=p_{\beta}^{\prime}$,
(B) $p_{\alpha}<p_{\beta}$ iff $p_{\alpha}^{\prime}<p_{\beta}^{\prime}$.

Proof of (A). Suppose $p_{\alpha}=p_{\beta}$. Then $i_{\alpha-1}=i_{\beta-1}$ and $i_{\alpha}=i_{\beta}$. Moreover, $k_{\alpha-1}=k_{\beta-1}$ in view of (2.15). Therefore, $i_{\alpha-1}^{\prime}=i_{\beta-1}^{\prime}, i_{\alpha}^{\prime}=i_{\beta}^{\prime}$, and $k_{\alpha-1}^{\prime}=$ $k_{\beta-1}^{\prime}$. Since $p p_{\alpha}=p p_{\beta}$, we have $r r_{\alpha}=r r_{\beta}$ and $s s_{\alpha}=s s_{\beta}$ by (2.18). Therefore, $r r_{\alpha}^{\prime}=r r_{\beta}^{\prime}$ and $s s_{\alpha}^{\prime}=s s_{\beta}^{\prime}$. This gives $p p_{\alpha}^{\prime}=p p_{\beta}^{\prime}$.

Proof of (B). Suppose $p_{\alpha}<p_{\beta}$. There are three ways this can occur.

(i) If $i_{\alpha-1}<i_{\beta-1}$, then $i_{\alpha-1}^{\prime}<i_{\beta-1}^{\prime}$ and so $p_{\alpha}^{\prime}<p_{\beta}^{\prime}$. 
(ii) If $i_{\alpha-1}=i_{\beta-1}$ and $i_{\alpha}<i_{\beta}$, then $i_{\alpha-1}^{\prime}=i_{\beta-1}^{\prime}$ and $i_{\alpha}^{\prime}<i_{\beta}^{\prime}$, so $p_{\alpha}^{\prime}<p_{\beta}^{\prime}$.

(iii) If $i_{\alpha-1}=i_{\beta-1}$ and $i_{\alpha}=i_{\beta}$ but $p p_{\alpha}<p p_{\beta}$, then $i_{\alpha-1}^{\prime}=i_{\beta-1}^{\prime}$ and $i_{\alpha}^{\prime}=i_{\beta}^{\prime}$. This case further breaks down into two possibilities. (a) If $k_{\alpha}<k_{\beta}$, then $k_{\alpha}^{\prime}<k_{\beta}^{\prime}$ and so $p_{\alpha}^{\prime}<p_{\beta}^{\prime}$. (b) $k_{\alpha}=k_{\beta}$ but $p p_{\alpha}<p p_{\beta}$. Therefore $\left(r r_{\alpha}, s s_{\alpha}\right)<\left(r r_{\beta}, s s_{\beta}\right)$. If $r r_{\alpha}<r r_{\beta}$, then $r r_{\alpha}^{\prime}<r r_{\beta}^{\prime}$ and so $p p_{\alpha}^{\prime}<p p_{\beta}^{\prime}$ by (2.18). If $r r_{\alpha}=r r_{\beta}$ and $s s_{\alpha}<s s_{\beta}$, then $r r_{\alpha}^{\prime}=r r_{\beta}^{\prime}$ and $s s_{\alpha}^{\prime}<s s_{\beta}^{\prime}$. So $p p_{\alpha}^{\prime}<p p_{\beta}^{\prime}$ by (2.18) again.

(2.35) Lemma. Fix $i=\left(i_{0}, \ldots, i_{m}\right)$ and $k=\left(k_{0}, \ldots, k_{m}\right)$ in the quadruple $\{i, k, r, s\}$. The number of elements in the order type of $\{i, k, r, s\}$ is a polynomial in the matrix entries $R\left(i_{\alpha}, k_{\alpha}\right)$ and $S\left(k_{\beta}, i_{\beta+1}\right)$ obtained by taking a product of some binomial coefficients

$$
\left(\begin{array}{c}
R\left(i_{\alpha}, k_{\alpha}\right) \\
a
\end{array}\right) \text { and }\left(\begin{array}{c}
S\left(k_{\beta}, i_{\beta+1}\right) \\
b
\end{array}\right)
$$

for certain $1 \leq a \leq m$ and $1 \leq b \leq m$. The form of the product only depends on $i, k$, and $m$.

Proof of (2.35). We start with a special case that illustrates the idea of the proof and is an ingredient in the general argument. Let $X=\{1,2, \ldots, n\}$ and let $\mathscr{L}$ be a finite set with $L$ elements. Let $f: X \rightarrow \mathscr{L}$ be a function having $d$ distinct values $v_{1}<\cdots<v_{d}$; let $v=\left\{v_{1}, \ldots, v_{d}\right\}$. Let $N_{f}$ denote the number of functions from $X$ to $\mathscr{L}$ having the same order type as $f$,

$$
N_{f}=\left(\begin{array}{l}
L \\
d
\end{array}\right)=\frac{L(L-1)(L-2) \cdots(L-d+1)}{d !} .
$$

In particular, $N_{f}=0$ whenever $L<d$. To prove (2.36), let $X_{i}=f^{-1}\left(v_{i}\right)$ for $1 \leq i \leq d$. Given any subset $w=\left\{w_{1}, \ldots, w_{d}\right\}$ of distinct values $w_{1}<\cdots<$ $w_{d}$ in $\mathscr{L}$, let $f_{w}: X \rightarrow \mathscr{L}$ be defined by $f_{w}(x)=w_{i}$ iff $x$ lies in $X_{i}$. Then $f_{v}=f$, each $f_{w}$ has the same order type as $f$, and the number of functions $f_{w}$ is precisely the binomial coefficient counting the number of subsets of $d$ elements in $\mathscr{L}$.

Since $i$ and $k$ are fixed, the number of elements in the order type of $\{i, k, r, s\}$ is the number $N_{r s}$ of elements in the order type of $\{r, s\}$. Moreover, $N_{r s}=N_{r} N_{s}$. We will show that $N_{r}$ is a product of certain binomial coefficients. The argument for $N_{s}$ is similar. Write the set $X=\{0,1, \ldots, m\}$ as the ordered disjoint union $X \stackrel{s}{=} X_{1} \cup \cdots \cup X_{c}$ where $\alpha$ and $\beta$ lie in the same $X_{i}$ iff $\left(i_{\alpha}, k_{\alpha}\right)=\left(i_{\beta}, k_{\beta}\right)$ and the order of the indices $u$ of the sets $X_{u}$ is such that $u<v$ if and only if $\left(i_{\alpha}, k_{\alpha}\right)<\left(i_{\beta}, k_{\beta}\right)$ for $\alpha$ in $X_{u}$ and $\beta$ in $X_{v}$. Note that $u<v$ implies $r_{\alpha}<r_{\beta}$ for $\alpha$ in $X_{u}$ and $\beta$ in $X_{v}$. It follows that $N_{r}=\prod_{u} N_{u}$ where $N_{u}$ is the number of elements in the order type of the subsequence of $r$ consisting of those $r_{\alpha}$ with $\alpha$ belonging to $X_{u}$. If both $r_{\alpha}$ and $r_{\beta}$ have $\alpha$ and $\beta$ in $X_{u}$, then $r_{\alpha}<r_{\beta}$ iff $r r_{\alpha}<r r_{\beta}$. Therefore $N_{u}$ is given by a binomial coefficient as in (2.36) where $L=R\left(i_{\alpha}, k_{\alpha}\right)$ and $1 \leq d \leq m$. 
To prove (2.31) we will show separately that $\mathrm{GY}_{m}(R, S)$ and $\mathrm{OS}_{q}(R, S)$ for $q$ dividing $m$ are given by sums of products of certain binomial coefficients. There are three contributions to $\mathrm{GY}_{m}(R, S)$ and $\operatorname{OS}_{q}(R, S)$ coming from Steps 1, 2, and 3. The proof for each of these steps is similar. So we just give the argument for Step 2.

The gyration number. Consider a periodic point $\{i, k, r, s\}$ of period exactly $m$ that is the least lexicographical element in its orbit. In Step 2 this goes to $\{k, i, s, \sigma(r)\}$ and the contribution to the gyration number is the rotation number of $\{k, i, s, \sigma(r)\}$, which only depends on the order type of $\{i, k, r, s\}$ by (2.33). By (2.34) the order type of $\{k, i, s, \sigma(r)\}$ is determined by the order type of $\{i, k, r, s\}$. In view of (2.35) we get the binomial coefficient formula for counting gyration numbers by observing that $\mathrm{GY}_{m}(R, S)$ is given by

(1) the sum over $p$ where $0 \leq p \leq m-1$ of

(2) $p$ times the sum over $\{i, k\}$ where $\{i, k\}$ is the least lexicographical element in its $\sigma$-orbit of

(3) the sum over $\{r, s\}$ so that

(a) period $\{i, k, r, s\}=m$ and $\{i, k, r, s\}$ is the least lexicographical element in its $\sigma$-orbit and

(b) the rotation number of $\{i, k, r, s\}$ is $p$.

The orbit-sign number. We must count the number of pairs $\{(i, k, r, s\}$, $\left.\left\{i^{\prime}, k^{\prime}, r^{\prime}, s^{\prime}\right)\right\}$ of period $q$ quadruples of least lexicographical order in their orbits so that $\{i, k, r, s\}<\left\{i^{\prime}, k^{\prime}, r^{\prime}, s^{\prime}\right\}$ and so that the least elements in the orbits of $\{k, i, s, \sigma(r)\}$ and $\left\{k^{\prime}, i^{\prime}, s^{\prime}, \sigma\left(r^{\prime}\right)\right\}$ respectively have the reverse order. If $i=\left\{i_{0}, \ldots, i_{m}\right\}$ and $i^{\prime}=\left\{i_{0}^{\prime}, \ldots, i_{m}^{\prime}\right\}$ where $i_{0}=i_{m}$ and $i_{0}^{\prime}=i_{m}^{\prime}$, let $i i^{\prime}=\left\{i_{0}, \ldots, i_{m}, i_{0}^{\prime} \ldots, i_{m}^{\prime}\right\}$. Similarly for $k k^{\prime}, r r^{\prime}, s s^{\prime}$. All these concatenated sequences each have at most $2 m$ distinct values. The order type of $\left\{i i^{\prime}, k k^{\prime}, r r^{\prime}, s s^{\prime}\right\}$ determines the order type of $\{i, k, r, s\}$ and of $\left\{i^{\prime}, k^{\prime}\right.$, $\left.r^{\prime}, s^{\prime}\right\}$, their periods, etc., and whether or not $\{i, k, r, s\}<\left\{i^{\prime}, k^{\prime}, r^{\prime}, s^{\prime}\right\}$. It also determines the order type of $\left\{k k^{\prime}, i i^{\prime}, s s^{\prime}, \sigma(r) \sigma\left(r^{\prime}\right)\right\}$, which in turn determines whether the order is reversed between the least element in the orbit of $\{k, i, s, \sigma(r)\}$ and the least element of $\left\{k^{\prime}, i^{\prime}, s^{\prime}, \sigma\left(r^{\prime}\right)\right\}$. As in the gyration number argument, we apply (2.33), (2.34), and (2.35) to get the binomial coefficient formula for orbit-sign numbers by observing that $\operatorname{OS}_{q}(R, S)$ is

(1) the sum over $\left\{i i^{\prime}, k k^{\prime}\right\}$ where $\{i, k\}$ and $\left\{i^{\prime}, k^{\prime}\right\}$ are the least lexicographical elements in their orbits of

(2) the sum over $\left\{r r^{\prime}, s s^{\prime}\right\}$ so that

(a) period $\{i, k, r, s\}=\operatorname{period}\left\{i^{\prime}, k^{\prime}, r^{\prime}, s^{\prime}\right\}=q$,

(b) $\{i, k, r, s\}$ and $\left\{i^{\prime}, k^{\prime}, r^{\prime}, s^{\prime}\right\}$ are the least lexicographical element in their orbits,

(c) $\{i, k, r, s\}<\left\{i^{\prime}, k^{\prime}, r^{\prime}, s^{\prime}\right\}$ but the least element in the orbit of $\{k, i, s, \sigma(r)\}$ is greater than the least element in the orbit of $\left\{k^{\prime}, i^{\prime}, s^{\prime}, \sigma\left(r^{\prime}\right)\right\}$.

This completes the proof of (2.31). 


\section{Construction of $\operatorname{sgcc}_{A, m}$}

We will use the isomorphism

$$
\operatorname{Aut}\left(s_{A}\right) \approx \pi_{1}(R S(Z), A)
$$

established in [W4] to obtain $\operatorname{sgcc}_{A, m}$. The idea is to start with a loop

$$
\gamma=\prod_{i} \gamma\left(R_{i}, S_{i}\right)^{\epsilon_{i}}
$$

in $R S(Z)$ based at $A$ where $\epsilon_{i}= \pm 1$ and $\left(R_{i}, S_{i}\right): P(i-1) \rightarrow P(i)$ or $\left(R_{i}, S_{i}\right): P(i) \rightarrow P(i-1)$ depending on whether $\epsilon_{i}=+1$ or $\epsilon_{i}=-1$, respectively. This will then be modified to a new "nonnegative" loop called $\gamma p(\gamma)$ in $R S\left(Z^{+}\right)$for a certain conveniently chosen polynomial $p(x)=c_{0}+c_{1} x+\cdots+c_{t} x^{t}$ where each $c_{i}$ is a nonnegative integer. We will define

$$
\operatorname{sgcc}_{A, m}(\gamma)=\operatorname{SGCC}_{A, m}(\gamma p(\gamma))
$$

and show that (3.2) is independent of the choices involved in picking $p(x)$ and under any deformation of $\gamma$ using the Triangle Identities in $R S(Z)$. Using (2.9), it will follow directly from the construction that $\operatorname{sgcc}_{A, m}$ is a homomorphism and that $\operatorname{SGCC}_{A, m}(\gamma)=\operatorname{sgcc}_{A, m} \cdot \delta_{A}(\gamma)$ whenever $A$ is nonnegative and aperiodic and $\gamma$ is nonnegative.

(3.3) Observation. The main point of this section is to verify that (3.2) gives a well-defined homomorphism $\operatorname{sgcc}_{A, m}$ on $\operatorname{Aut}\left(s_{A}\right) \approx \pi_{1}(R S(Z), A)$. But once this is done, the proof also shows how to compute $\operatorname{sgcc}_{A, m}(\gamma)$ directly as follows: if necessary, replace $R_{i}$ by $R_{i} \operatorname{sgn}\left(R_{i}\right)$ and $S_{i}$ by $S_{i} \operatorname{sgn}\left(S_{i}\right)$ as in (3.5) and (3.7) to make the signs of $R_{i}$ and $S_{i}$ equal to +1 . Then apply the algebraic formula (2.14) when $m=2$ or the more complicated (2.31) when $m>2$ to each edge $\left(R_{i}, S_{i}\right)$ in (3.1) and multiply the result by $\epsilon_{i}= \pm 1$. Finally, sum up over the edges. This is the method used for the examples in $\S 4$.

We need two preliminary lemmas.

(3.4) Lemma. Let $\Lambda$ be any dense unital subring of the real numbers $\mathbf{R}$. Let $M$ be a square matrix over $\Lambda$ that is algebraically shift equivalent over the complex numbers to an eventually positive real matrix. Then $M$ is conjugate over $\Lambda$ to an eventually positive matrix.

This is due to Handelman [H, Theorem 2.3]. The result in $[\mathrm{H}]$ is stated for $\Lambda=Q$, but the proof works in the same manner for any $\Lambda$ as in (3.4).

Let $R: P \rightarrow Q$ be a shift equivalence over $\mathbf{R}$ between two eventually positive matrices $P$ and $Q$ over $\mathbf{R}$. Choose positive right eigenvectors $v_{P}$ and $v_{Q}$ for $P$ and $Q$, respectively, corresponding to the Perron-Frobenius eigenvalue. Then $R v_{Q}=\alpha v_{P}$ for some nonzero scalar $\alpha$, and we define

$$
\operatorname{sgn}(R)= \begin{cases}+1 & \text { if } \alpha>0, \\ -1 & \text { if } \alpha<0\end{cases}
$$

If $R_{1}: M \rightarrow P$ and $R_{2}: P \rightarrow Q$ are shift equivalences between eventually positive matrices, then

$$
\operatorname{sgn}\left(R_{1} R_{2}\right)=\operatorname{sgn}\left(R_{1}\right) \operatorname{sgn}\left(R_{2}\right) .
$$

The following lemma is well known. See [KR1; PW, p. 493]. 
(3.7) Lemma. Let $R: P \rightarrow Q$ be a shift equivalence over $\mathbf{R}$ between two eventually positive matrices $P$ and $Q$. There is a positive integer $n$ so that for all $k \geq n$ the matrices $P^{k}$ and $Q^{k}$ are positive and the matrix $R Q^{k}$ is positive if $\operatorname{sgn}(R)=+1$ and negative if $\operatorname{sgn}(R)=-1$.

Observe that if the edge $(R, S): P \rightarrow Q$ is a strong shift equivalence between eventually positive matrices $P$ and $Q$, then $\operatorname{sgn} R=\operatorname{sgn} S$. If $\operatorname{sgn}(R)=$ $\operatorname{sgn}(S)=+1$, we say that the edge is eventually positive.

Construction of $\gamma p(\gamma)$. Let $\Lambda$ denote the ring of all rational numbers that can be written in the form $a / b$ where $b$ is congruent to $1 \bmod m((2 m) !)$. The integers $a$ and $b$ need not be relatively prime.

Step 1. For each vertex $P$ in the loop $\gamma$, use (3.4) to choose a conjugacy $C$ over $\Lambda$ between $P$ and an eventually positive matrix $C P C^{-1}$. A typical edge $(R, S): P \rightarrow Q$ in $\gamma$ is then replaced by the edge

$$
\left(C R D^{-1}, D S C^{-1}\right): C P C^{-1} \rightarrow D Q D^{-1},
$$

where now both $C P C^{-1}$ and $D Q D^{-1}$ are eventually positive. There is the following diagram in $R S(\Lambda)$ :

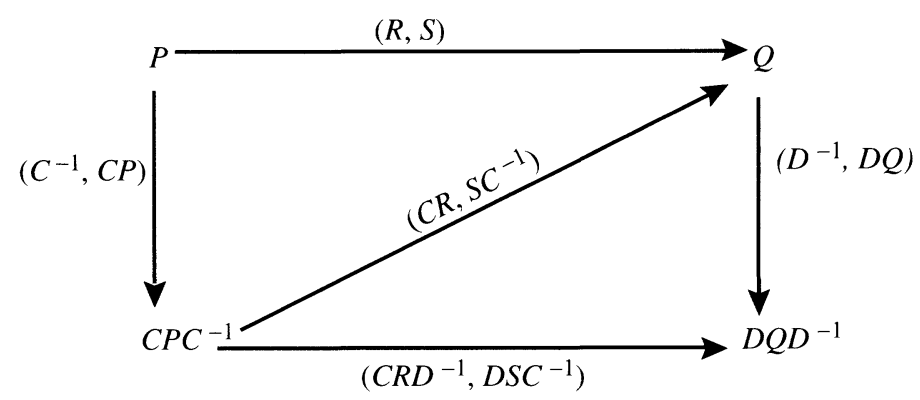

This provides a homotopy in $R S(\Lambda)$ from the original loop $\gamma$ to a loop where the vertices are all eventually positive. By replacing each $(E, F): M \rightarrow N$ in this loop by the edge $(E \operatorname{sgn}(E), F \operatorname{sgn}(F)): M \rightarrow N$, we obtain a new loop $\gamma$ with the property that all its edges are eventually positive.

Step 2. Now apply (3.7) to find a large positive integer $n$ so that for all edges $(E, F): M \rightarrow N$ in the new eventually positive loop $\gamma$, the matrices $M^{k}, N^{k}, E N^{k}$, and $F M^{k}$ are positive for $k \geq n$. Fix integers $k \geq n$ and $l \geq n$. Let $a(x)=a_{0}+a_{k} x^{k}$ and $b(x)=b_{0}+b_{l} x^{l}$ be polynomials with strictly positive rational coefficients $a_{i}$ and $b_{j}$ satisfying

(i) both the numerators and the denominators of $a_{0}$ and $b_{0}$ are congruent to $1 \bmod m((2 m) !)$,

(ii) the numerators of $a_{k}$ and $b_{k}$ are congruent to $0 \bmod m((2 m) !)$ and the denominators of $a_{k}$ and $b_{k}$ are congruent to $1 \bmod m((2 m) !)$.

Next choose the $a_{i}$ and $b_{j}$ in (i) and (ii) so that the polynomials $a(x)$ and $b(x)$ are sufficiently good approximations to $x^{k}$ and $x^{l}$ respectively to ensure 
(iii) Let $g(x)=a(x) b(x)$. Then for all edges $(E, F): M \rightarrow N$ in the new loop $\gamma$, the matrices $M g(M), N g(N), E a(N)$, and $F b(M)$ are positive.

Now let $D>0$ be the product of the denominators of the coefficients of $a(x)$ and $b(x)$ and of all the denominators occurring in the entries of the positive matrices $M f(M), N f(N), E a(N)$, and $F b(M)$ of (iii) where $(E, F): M \rightarrow$ $N$ runs through all the edges of $\gamma$. Let $r(x)=D a(x), s(x)=D b(x)$, and $p(x)=r(x) s(x)$.

(iv) The polynomials $p(x), r(x)$, and $s(x)$ are positive, integral, and congruent to $1 \bmod m((2 m) !)$. Moreover, for all edges $(E, F): M \rightarrow N$ in the loop $\gamma$, the matrices $M p(M), N p(N), \operatorname{Er}(N)$, and $F s(M)$ are positive and integral.

We let $\gamma p(\gamma)$ denote the loop in $R S\left(Z^{+}\right)$obtained by replacing each edge $(E, F): M \rightarrow N$ in the new loop $\gamma$ with the edge

$$
(\operatorname{Er}(N), F s(M)): M p(M) \rightarrow N p(N) .
$$

Step 3. Finally, we define $\operatorname{sgcc}_{m}$ as in (3.2) and see from (2.9) that it is a sum of terms of the form

$$
\pm \mathrm{SGCC}_{m}(\operatorname{Er}(N), F s(M))
$$

where $\mathrm{SGCC}_{m}$ is computed with respect to the standard lexicographical $m$ basis at each vertex in the loop $\gamma p(\gamma)$.

Proof that $\operatorname{sgcc}_{A, m}(\gamma p(\gamma))$ is well defined. Let $(E, F): M \rightarrow N$ be any strong shift equivalence over $\Lambda$. Let $r(x)$ and $s(x)$ be a pair of polynomials satisfying the property (iv) of Step 2. Let $e(x)$ and $f(x)$ be another such pair. From (2.30) we have

$$
\begin{aligned}
\operatorname{SGCC}_{m}(\operatorname{Er}(N), F s(M)) & =\operatorname{SGCC}_{m}(E r(N) e(N), F s(M) f(M)) \\
& =\operatorname{SGCC}_{m}(E e(N), F f(M)) .
\end{aligned}
$$

This shows that the value of $\operatorname{sgcc}_{m}$ on a loop whose edges are already eventually positive is independent of the choice of polynomials $r(x)$ and $s(x)$ made in Step 2.

Next we show $\operatorname{sgcc}_{m}$ does not depend on the choices of vertex conjugacies made in Step 1. Suppose a first choice of vertex conjugacies gives a loop $\alpha$ as in Step 1 and another choice gives a loop $\beta$. These two conjugacies combine to give a conjugacy from each vertex of $\alpha$ to the corresponding vertex of $\beta$ and to produce a one step homotopy from $\alpha$ to $\beta$ in which a typical rectangle has the form (3.8). The loop edge across the top of (3.8) is now an edge of $\alpha$, and the one on the bottom is the corresponding edge of $\beta$. Replace each edge $(E, F): M \rightarrow N$ in the homotopy with the edge $(E \operatorname{sgn}(E), F \operatorname{sgn}(F)): M \rightarrow$ $N$. This preserves the Triangle Identities. We obtain a homotopy from the two end results $\alpha$ and $\beta$ of Step 1 where now each edge in the homotopy is eventually positive. At this point choose polynomials $r(x)$ and $s(x)$ so that property (iv) of Step 2 is satisfied for all edges in the homotopy. We then replace this first choice of $r(x), s(x)$, and $p(x)$ by new polynomials $r(x)=p(x)^{2}$, $s(x)=p(x)^{2}$, and $p(x)=p(x)^{4}$ and obtain a homotopy in $R S\left(Z^{+}\right)$from $\alpha p(\alpha)$ to $\beta p(\beta)$ in which a typical rectangle is 


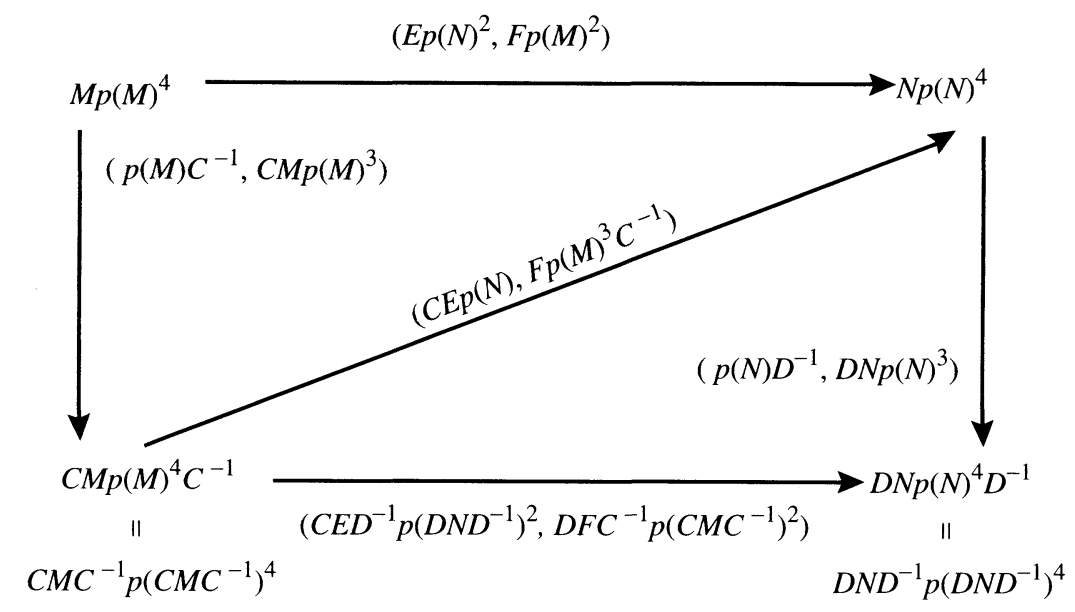

Hence $\operatorname{sgcc}_{m}(\alpha p(\alpha))=\operatorname{sgcc}_{m}(\beta p(\beta))$, because $\operatorname{SGCC}_{A, m}$ is well defined on $\pi_{1}\left(R S\left(Z^{+}\right), A\right)$.

Finally, we show $\operatorname{sgcc}_{m}$ is independent of any deformation of $\gamma$ by Triangle Identities in $R S(Z)$. Consider the triangle

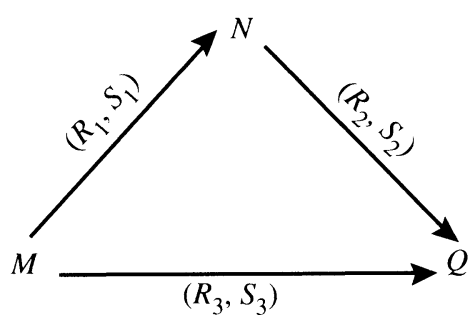

in the component of $R S(Z)$ containing $A$. As above we choose a conjugacy over $\Lambda$ at each vertex to make each of the vertices $M, N$, and $Q$ eventually positive. This triangle then becomes

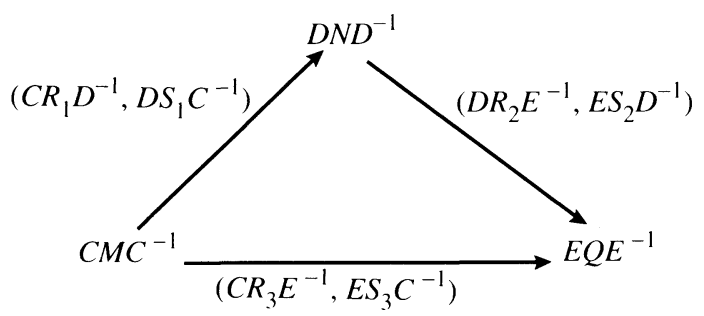

Also, replace each edge $(R, S)$ in this triangle with the edge $(R \operatorname{sgn}(R)$, $S \operatorname{sgn}(S))$. In effect, we can assume that the triangle (3.11) has eventually positive vertices and edges. Choose polynomials $r(x), s(x)$, and $p(x)=r(x) s(x)$ so that property (iv) of Step 2 is satisfied for all edges in the homotopy. We then replace this first choice of $r(x), s(x)$, and $p(x)$ by new polynomials $r(x)=$ 
$p(x), s(x)=p(x)^{2}$, and $p(x)=p(x)^{3}$ and obtain the following triangle in $R S\left(Z^{+}\right)$:

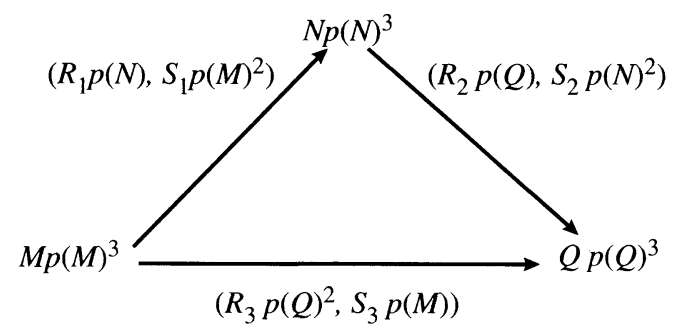

This implies

$$
\begin{aligned}
\operatorname{sgcc}_{m}\left(R_{3} p(Q)^{2}, S_{3} p(M)\right)= & \operatorname{sgcc}_{m}\left(R_{1} p(N), S_{1} p(M)^{2}\right) \\
& +\operatorname{sgcc}_{m}\left(R_{2} p(Q), S_{2} p(N)^{2}\right) .
\end{aligned}
$$

We are now done with the construction of $\operatorname{sgcc}_{A, m}$ and the proof of (1.4).

The last result in this section discusses the relation between $\operatorname{sgcc}_{A}$ and $\operatorname{sgcc}_{B}$ when $A$ and $B$ are as in (1.4) and lie in the same path component of $S(Z)$, i.e., $A$ and $B$ are shift equivalent over $Z$. By [W4] we know that $A$ and $B$ lie in the same path component of $R S(Z)$. Choose a path $\alpha$ from $B$ to $A$ in $R S(Z)$. Define an isomorphism $\alpha_{*}$ from $\operatorname{Aut}\left(s_{A}\right)$ to $\operatorname{Aut}\left(s_{B}\right)$ by letting $\alpha_{*}(\gamma)=\alpha \gamma \alpha^{-1}$ for each $\gamma$ in $\operatorname{Aut}\left(s_{A}\right)$. From $\S 2$ and the above discussion in $\S 3$, we then see that

$$
\operatorname{sgcc}_{A, m}=\operatorname{sgcc}_{B, m} \cdot \alpha_{*} \cdot
$$

\section{Applications of $\operatorname{sgcc}_{A, m}$}

(4.1) An example of a SFT where the dimension group representation is not surjective. Consider the $4 \times 4$ aperiodic matrix

$$
A=\left(\begin{array}{llll}
0 & 0 & 1 & 1 \\
1 & 0 & 0 & 0 \\
0 & 1 & 0 & 0 \\
0 & 0 & 1 & 0
\end{array}\right)
$$

Its characteristic polynomial is $t^{4}-t-1$. This yields the equation $(A-1)\left(A^{4}+\right.$ $\left.A^{3}+A^{2}\right)=A$ and the strong shift equivalence $A=R S=S R$ over $Z$ where $R=A-1$ and $S=A^{4}+A^{3}+A^{2}$. The matrix $R$ therefore represents an element of $\operatorname{Aut}\left(s_{A}\right)$. The construction of $\operatorname{sgcc}_{2}$ and the formula (2.14) gives $\operatorname{sgcc}_{2}(R)=1$ by direct computer computation. Using (1.4) we see that $R$ is not induced by an element in $\operatorname{Aut}\left(\sigma_{A}\right)$, because there are no fixed points or orbits of period 2. From (3.12) we see that the dimension group representation is also not surjective for any aperiodic matrix that is shift equivalent to $A$ over $Z$. This example is used in [KR3] to give examples of subshifts of finite type (reducible, with two aperiodic components) that are shift equivalent but not strong shift equivalent over the nonnegative integers. 
(4.2) An example of an automorphism of the period 6 points of the full Bernoulli 2-shift that is not induced by an element in $\operatorname{Aut}\left(\sigma_{A}\right)$. This example was first suggested by U. Fiebig and confirmed using results in [KR2]. We give an alternate proof here. Let $\alpha$ be an automorphism of the 2-shift restricted to the period 6 points that shifts one orbit to the left once and leaves all others fixed. Then $\operatorname{SGCC}_{6}(\alpha)=1 \bmod (6)$. On the other hand, $\operatorname{Aut}\left(s_{A}\right)=Z$ and is generated by $s_{2}$. But $\operatorname{sgcc}_{6}\left(s_{2}\right)=3 \bmod (6)$. By (1.4) the image of Aut $\left(\sigma_{A}\right)$ under $\operatorname{SGCC}_{6}$ is therefore the subgroup of index 2 in $Z / 6$ generated by 3 . Hence, $\alpha$ does not come from an automorphism of the shift.

(4.3) An example of a subshift of finite type with a permutation of fixed points that is not induced by an element in $\operatorname{Aut}\left(\sigma_{A}\right)$. Consider the $3 \times 3$ aperiodic matrix

$$
A=\left(\begin{array}{lll}
1 & 1 & 0 \\
0 & 1 & 1 \\
5 & 0 & 0
\end{array}\right)
$$

The shift corresponding to $A$ has exactly two fixed points and no period two orbits. If the transposition of the fixed points could be lifted to an automorphism $\alpha$ in $\operatorname{Aut}\left(\sigma_{A}\right)$ then we would have $\operatorname{SGCC}_{2}(\alpha)=1$. We will show this cannot happen by verifying that $\operatorname{sgcc}_{2}$ vanishes identically on the order preserving elements of $\operatorname{Aut}\left(s_{A}\right)$ and then applying (1.4).

The characteristic polynomial of $A$ is $\chi(t)=t^{3}-2 t^{2}+t-5$. This has no rational roots, and it is therefore irreducible over $Q$. Let $\lambda$ be the PerronFrobenius eigenvalue. A positive right eigenvector for $\lambda$ is $v_{\lambda}=(1, \lambda-1$, $\left.(\lambda-1)^{2}\right)$. The correspondence sending $u$ in $Z^{3}$ to the dot product $u v_{\lambda}$ induces an isomorphism between the dimension group $G(A)$ and the subring $Z[1 / \lambda]$ of the real numbers under which the action of $A$ goes to multiplication by $\lambda$. Moreover, the order-preserving elements of $\operatorname{Aut}\left(s_{A}\right)$ are identified with the group of the positive units in $Z[1 / \lambda]$. See [BLR, 6.4]. Using the usual discriminant formula [We, p. 105], we see that $\Delta\left(1, \lambda, \lambda^{2}\right)=-655$. This is squarefree, so $Z[\lambda]$ is the ring of integers in $Q(\lambda)$. The principal ideal generated by $\lambda$ is prime because its norm is the rational prime 5 . The polynomial $t^{3}-2 t^{2}+t-5$ has one real root and two complex ones. Using the $S$-unit theorem [We, 5-3-10], we deduce that the group of positive units in $Z[1 / \lambda]$ is free abelian of rank two isomorphic to the direct sum of infinite cyclic group generated by $\lambda$ and infinite cyclic group $P$ of positive units in $Z[\lambda]$.

We know that $\operatorname{sgcc}_{2}(\lambda)=0$ because $\lambda$ is the isomorphism induced on the dimension group by the shift $\sigma_{A}$ that does not move the fixed points. It remains to show $\operatorname{sgcc}_{2}$ vanishes on $P$. Since $\operatorname{sgcc}_{2}$ takes values in $Z / 2$, it will be sufficient to find an element $\alpha$ of $P$ that is not a square (in $\mathrm{P}$ ) and so that $\operatorname{sgcc}_{2}(\alpha)=0$. A direct computer search found the following equation in $Z[\lambda]$ :

$$
\left(4+\lambda+2 \lambda^{2}\right)\left(\lambda^{2}-2 \lambda-1\right)=1 .
$$

We claim $\alpha=4+\lambda+2 \lambda^{2}$ has the desired properties. $Z[\lambda]$ is isomorphic to the quotient of $Z[t]$ by the ideal generated by $\chi(t)$. We have $\chi(2)=-3$. So evaluation at $t=2$ yields a homomorphism of $Z[\lambda]$ to $Z / 3$ under which $\alpha$ 
goes to 2 . Since 2 is not a square modulo $3, \alpha$ cannot be a square in $P$. To compute $\operatorname{sgcc}_{2}(\alpha)$, observe that (4.4) gives the matrix equation

$$
\left(4+A+2 A^{2}\right)\left(A^{3}-2 A^{2}-A\right)=A .
$$

The action of $\alpha$ on the dimension group therefore comes from the strong shift equivalence $(R, S): A \rightarrow A$ over $Z$ where $R=4+A+2 A^{2}$ and $S=A^{3}-$ $2 A^{2}-A$. The construction of $\operatorname{sgcc}_{2}$ and the formula (2.14) gives $\operatorname{sgcc}_{2}(\alpha)=$ $\operatorname{sgcc}_{2}(R)=0$ by direct computer computation.

(4.5) An example where surjectivity of the dimension group representation in a certain case would imply that FOG is false. Example (4.1) shows that in general the dimension group representation $\delta_{A}: \operatorname{Aut}\left(\sigma_{A}\right) \rightarrow \operatorname{Aut}\left(s_{A}\right)$ is not surjective. The finite order generation problem FOG asks whether every element in the kernel of the dimension group representation $\delta_{A}: \operatorname{Aut}\left(\sigma_{A}\right) \rightarrow \operatorname{Aut}\left(s_{A}\right)$ is a product of elements of finite order. The example below is given to show the possible close relation between FOG and the question of whether $\delta_{A}$ is surjective.

Let $A$ be the matrix in (4.1) and let $B=A^{3}$. The characteristic equation $t^{4}-t-1=0$ for $A$ gives the matrix equations

$$
(A-1)\left(A^{3}+A^{2}+A\right)=1 \quad \text { and }(A-1)\left(B A^{3}+B A^{2}+B A\right)=B .
$$

Let $(R, S): B \rightarrow B$ where $R=A-1$ and $S=B A^{3}+B A^{2}+B A$. As in the previous examples, we see that $R$ induces an automorphism of the dimension group $G_{B}$ and that $\operatorname{sgcc}_{B, 2}(R)=1$.

Suppose $\delta_{B}$ is surjective. Let $\beta$ be any element of $\operatorname{Aut}\left(\sigma_{B}\right)$ that induces the same automorphism of $G_{B}$ as does $R$. By (1.4) we would have $\operatorname{SGCC}_{B, 2}(\beta)=$ 1. This implies $\beta$ must switch two fixed points and leave the other one fixed. The elementary conjugacy $\alpha$ corresponding to the strong shift equivalence $\left(A^{2}, A\right): B \rightarrow B$ is the cyclic permutation (123) on the fixed points. We always get $\alpha \beta \alpha^{-1} \beta^{-1}=(132)$ on fixed points for $\beta=(12)$, (13), or (23). In this case $\operatorname{Aut}\left(s_{B}\right)$ is abelian [BLR, 6.5], and therefore $\alpha \beta \alpha^{-1} \beta^{-1}$ is inert. But the argument of [BLR, 7.3] applies also in this case to show that any element of $\operatorname{Aut}\left(\sigma_{B}\right)$ that is a product of elements of finite order must induce the identity on fixed points. Hence FOG would fail for $\alpha \beta \alpha^{-1} \beta^{-1}$.

\section{ACKNOWLEDGMENT}

We would like to thank Mike Boyle and David Handelman for conversations about this material. We are also very grateful to the referee for a careful reading of the manuscript that improved the final version.

\section{REFERENCES}

[B] K. Baker, Strong shift equivalence of 2 by 2 matrices of non negative integers, Ergodic Theory Dynamical Systems 3 (1983), 501-508.

[BH] M. Boyle and D. Handelman, Algebraic shift equivalence and primitive matrices, preprint, Univ. of Maryland 1990.

[BK] M. Boyle and W. Krieger, Periodic points and automorphisms of the shift, Trans. Amer. Math. Soc. 302 (1987), 125-149. 
[BLR] M. Boyle, D. Lind, and D. Rudolph, The automorphism group of a shift of finite type, Trans. Amer. Math. Soc. 306 (1988), 71-114.

[CK] J. Cuntz and W. Krieger, Topological Markov chains and dicyclic dimension groups, J. Reine Angew. Math. 320 (1980), 44-51.

[DGS] M. Denker, C. Grillenberger, and K. Sigmund, Ergodic theory on compact spaces, Lecture Notes in Math, vol. 527, Springer-Verlag, Berlin-Heidelberg-New York, 1976.

[E] E. Effros, Dimensions and $C^{*}$-algebras, CBMS, No. 46, Amer. Math. Soc., Providence, RI, 1981.

[F1] U. Fiebig, Uber gyrationszahlenfolgen und ein darstellungenproblem in der symbolischen dynamik, Dissertation, Univ. of Gottingen, 1987.

[F2] _ Gyration numbers for involutions of subshifts of finite type, Math. Forum (to appear).

[Fr] J. Franks, Homology and dynamical systems, CBMS No. 49, Amer. Math. Soc., Providence, RI, 1982.

[H] D. Handelman, Positive matrices and dimension groups, J. Operator Theory 6 (1981), 5574.

[He] G. Hedlund, Endomorphisms and automorphisms of shift dynamical systems, Math. Systems Theory 3 (1969), 320-375.

[K] W. Krieger, On dimension functions and topological Markov chains, Invent. Math. 56 (1980), 239-250.

[KR1] H. Kim and F. W. Roush, Some results on decidability of shift equivalence, J. Combin. Inform. System Sci. 4 (1979), 123-146.

[KR2] _ On the structure of inert automorphisms of subshifts, preprint, Alabama State Univ. at Montgomery 1989.

[KR3] _ Williams's conjecture is false for reducible subshifts, J. Amer. Math. Soc. 5 (1992), 213-215 (this issue).

[N] M. Nasu, Topological conjugacy for sofic systems and extensions of automorphisms of finite subsystems of topological Markovc chaings, Dynamical Systems, Lecture Notes in Math., vol. 1342, Springer-Verlag, Berlin-Heidelberg-New York, 1988, pp. 564-607.

[PT] W. Parry and S. Tuncel, Classification problems in ergodic theory, LMS Lecture Notes, No. 67, Cambridge Univ. Press, 1982.

[PW] W. Parry and R. F. Williams, Block coding and a zeta function for finite Markov chains, Proc. London Math. Soc. (3) 35 (1977), 483-495.

[W1] J. Wagoner, Markov partitions and $K_{2}$, Publ. Math. IHES, no. 65, 1987.

[W2] _ Triangle Identities and symmetries of a subshift of finite type, Pacific J. Math. 144 (1990), 181-205.

[W3] _ Eventual finite order generation for the kernel of the dimension group representation, Trans. Amer. Math. Soc. 317 (1990), 331-350.

[W4] _ Higher dimensional shift equivalence is the same as strong shift equivalence over the integers, Proc. Amer. Math. Soc. 109 (1990), 527-536.

[We] E. Weiss, Algebraic number theory, McGraw-Hill, New York, 1963.

[Wi1] R. F. Williams, Classification of one-dimensional attractors, Proc. Sympos. Pure Math. vol. 14, Amer. Math. Soc., Providence, RI, 1970, pp. 341-361.

[Wi2] __ Classification of subshifts of finite type, Ann. of Math. (2) 98 (1973), 120-153; Errata ibid. 99 (1974), 380-381.

(K. H. Kim and F. W. Roush) Department of Mathematics, Alabama State University, Montgomery, Alabama 36195

(J. B. Wagoner) Department of Mathematics, University of California, Berkeley, CalIFORNIA 94720 